\title{
USO DE LAS TIC EN LA GESTIÓN DEL CONOCIMIENTO EN LA UNIVERSIDAD \\ DEL ZULIA (NÚCLEO COL)
}

\section{USE OF THE ICT IN KNOWLEDGE MANAGEMENT AT UNIVERSIDAD DEL ZULIA (NUCLEO COL)}

\author{
Josmary del V. Rojas Oriquen* \\ Dubisay T. Morales Gutiérrez** \\ Carlos A. García Mora*** \\ Universidad de Zulia, Núcleo Costa Oriente del Lago
}

RECIBIDO: Agosto 12013

ACEPTADO: Octubre 52013

\section{RESUMEN}

El presente artículo producto de una investigación tiene como objetivo general evaluar

el uso de las TIC en la gestión del conocimiento en la Universidad del Zulia (Núcleo COL). La investigación es de diseño no experimental, de campo, y se realizó en el lugar de los acontecimientos. La población estuvo conformada por 4261 personas, distribuidos en estudiantes, docentes y personal administrativo de la Universidad del Zulia. El tamaño de la muestra es de trescientos sesenta y seis (366) estudiantes, treinta y seis (36) docentes y cuatro (4) representantes del personal administrativo. Como instrumento de recolección de datos, se utilizó una encuesta, la cual consta de

\footnotetext{
* Magister en telemática, Ingeniero de computación, vinculación institucional Universidad del Zulia, Venezuela, núcleo costa oriental del lago. Correo electrónico: josmaryrojas@gmail.com

** Dra. En Ciencias de la Educación, Magister en telemática, Ingeniero de Computación, Universidad del Zulia, núcleo costa oriental de lago. Correo electrónico: dubisay@gmail.com

*** Posdoctorado. En ciencias de la Educación, Decano de la Universidad del Zulia, núcleo costa oriental del lago. Correo electrónico: cagarcia1970@gmail.com
} 
26 ítems. Para la validez del contenido, se contó con el juicio de tres expertos y se obtuvo una confiabilidad 0,800. Con ayuda del sistema estadístico (SPSS 17), se obtuvieron los resultados, encontrándose que la población estudiantil y docente así como el personal administrativo implementan el uso de las TIC en la gestión del conocimiento como herramienta de enseñanza- aprendizaje, obteniendo así un proceso de educación productivo.

Palabras clave: TIC, gestión, conocimiento, tecnología de información. 


\begin{abstract}
The general objective of this research is to evaluate the use of the ICT in Knowledge Management at Universidad del Zulia (Nucleo COL). The research consists of a field trip activity of non experimental Design, carried out in the place of the events. The population is 4261 people including students, teachers and clerical staff of the Universidad del Zulia. The size of the sample is three hundred and sixty six (366) students, thirty six (36) teachers and four (4) individuals of the Clerical staff. A survey was the instrument used to gather information, which consists of 26 items. For the validity of the content, we counted on the opinion of three experts and we obtained a reliability of 0,800 . With the help of the statistical system (SPSS 17), the results show that students and teachers, as well as the clerical staff, implement the use of ICT in Knowledge management as a teaching-learning tool; this way, they achieved a productive educational process.
\end{abstract}

Key words: Management, Knowledge, Technology of Information 


\section{INTRODUCCIÓN}

Las nuevas tecnologías, aplicadas a una realidad en el proceso de enseñanza-aprendizaje, cumplen funciones básicas propias de los medios pedagógicos en general o pueden proporcionar funciones específicas, tales como el uso del Internet, correo electrónico, video conferencia, discusiones a través de la red, cursos on line, estudios a distancia, entre otros. Estas herramientas contribuyen a optimizar el proceso educativo, pues le brindan a docentes y alumnos opciones para una mejor comprensión de los objetivos establecidos en los programas de estudios.

Junto a las TIC aparece la gestión del conocimiento como una forma de asumir la creación/identificación, captura, organización, acceso, distribución y uso del conocimiento esencial en una institución. Los elementos claves en la gestión del conocimiento son las personas que forman la organización y los medios a su alcance para gestionar sus conocimientos, en este caso las TIC son esos elementos mediadores entre el aula y el estudiante. Por ello, en medio de las múltiples integraciones que demanda la dinámica transformadora y sobre todo ahora, cuando se asiste a tantos y tan complejos cambios simultáneos, la educación debe reconocer sus incongruencias, redefinir sus métodos, recompensar sus estructuras, de modo que se constituyan en fuentes de conocimiento nuevo y distinto. En este contexto, las nuevas tecnologías de telecomunicaciones juegan un papel clave, pues, transmiten la información y, lo que es más importante, la convierten en conocimiento. 
La universidad del Zulia (Núcleo COL) no escapa a esta realidad, y se ve en la necesidad de incorporar tecnología informática en las aulas, como mediadora de aprendizaje, lo cual permite a los agentes dinamizadores de procesos educativos en esta institución tener acceso de manera eficiente a la información en el campo del conocimiento y, a través de ello, tales agentes pueden sobrepasar las visiones y modelos que han orientando los procesos educativos de manera tradicional.

Es por ello que deben determinarse técnicas y métodos de análisis para el desarrollo de las TIC en la gestión del conocimiento, a partir de datos desarrollados bajo patrones de referencias comparativos como en el caso de este estudio, que permitirá establecer criterios y otros métodos alternativos. El avance de este tipo de proyectos servirá, así, como modelo a seguir para futuras investigaciones $\mathrm{y}$ contribuirá a la construcción del uso de las TIC en la educación venezolana.

\section{TECNOLOGÍAS DE INFORMACIÓN Y} COMUNICACIÓN (TIC)

Las TIC son quizá las herramientas tecnológicas más importantes del presente. Se necesitan para la gestión y transformación de la información, en particular en el uso de computadores y programas que permiten crear, modificar, almacenar, proteger $y$ recuperar esa información. En este sentido, Nishio (2004) establece que las tecnologías de información y comunicación comprenden a todas aquellas que permiten el manejo de información y facilitan diferentes formas 
de comunicación. El mismo autor plantea que las TIC se subdividen en tecnologías de captación, almacenaje, procesamiento, comunicación y visualización. Por su parte, Joyanes (1998) resalta la interactividad que se ha logrado a través de las tecnologías, y que la sociedad de la información capta, transporta, almacena, procesa y difunde información con rapidez. Desde esta perspectiva, se reconoce a las tecnologías de información de datos como una herramienta oportuna para la toma de decisiones. Estos puntos de vista son asumidos en la presente investigación, y permitirán establecer su vinculación con el otro asunto fundamental de ella: la gestión del conocimiento.

\section{GESTIÓN DEL CONOCIMIENTO}

La gestión del conocimiento comprende el proceso de planear, organizar, dirigir, evaluar, controlar y administrar continuamente conocimiento de todo tipo para satisfacer necesidades presentes y futuras, así como para identificar y explorar recursos de conocimiento tanto preexistentes como adquiridos y para desarrollar nuevas oportunidades.

A partir de la teoría basada en el conocimiento, los representantes de la escuela japonesa consideran que las organizaciones deben ser estudiadas según la forma cómo crean y transfieren conocimiento (Nonaka, 1994, Nonaka \& Takeuchi, 1995). No obstante debe tenerse claro que el concepto de gestión I conocimiento se puede definir de diferentes formas.

Para Brooking (1997), por ejemplo, la gestión del conocimiento es el área dedicada a la dirección de las tácticas y estrategias requeridas para la administración de los recursos 
intangibles humanos en una organización. En tal sentido, se presenta mediante perspectivas tácticas y operativas, es más detallada que la gestión del capital intelectual y se centra en la forma de dar a conocer $y$ administrar las actividades relacionadas con el conocimiento, como su creación, captura, transformación y uso. Su función es planificar, implementar y controlar todas las actividades relacionadas con el conocimiento y los programas requeridos para la administración efectiva del capital intelectual.

Por su parte, Bueno (1999) señala que la gestión del conocimiento es la función que planifica, coordina y controla los flujos de conocimiento que se producen en la empresa en relación con sus actividades y su entorno, con el fin de crear unas competencias esenciales.
Sobre la base de las ideas expuestas, se puede decir que, en el contexto de las universidades, la gestión del conocimiento puede entenderse como un proceso que implica la propagación de nuevas ideas y la transferencia de conocimiento mediante la integración de herramientas tecnológicas.

\section{MÉTODO}

Esta investigación es de campo, de tipo descriptiva, de diseño no experimental y se apoya en las teorías de Bavaresco (2001) y Tamayo \& Tamayo (2001). En ella se analiza una situación actual que se presenta en las instalaciones de la Facultad de Ingeniería de la Universidad del Zulia (Núcleo COL) en cuanto al uso adecuado o inadecuado de herramientas tecnológicas de 
información y comunicación en la

gestión del conocimiento, describiendo

un entorno para luego evaluar sus

características, a través del

establecimiento de la hipótesis

descriptiva. Según Bavaresco (2001,

p.31), el diseño no experimental

consiste en la recolección de datos

directamente de la realidad de donde

ocurren los hechos, sin manipular o

controlar variable alguna. El diseño de la

presente investigación es no

experimental, porque aun cuando se

analiza una realidad presente en el

programa de Ingeniería de la

Universidad del Zulia (Núcleo COL), a

través de técnicas de recolección de datos, y se evalúan las variables de estudios como la gestión de conocimiento y tecnologías de información y comunicación, no se ejerce ningún tipo de manipulación sobre dichas variables.

\section{Población y muestra}

Para el desarrollo de esta investigación, se trabajó con una población que comprendía 4301 sujetos del programa de Ingeniería de la Universidad del Zulia (Núcleo COL), de los cuales 36 son docentes, 4 pertenecen al rango administrativo y 4261 son estudiantes, como se observa en el Cuadro 01:

Cuadro 01. Población Total en la Universidad del Zulia (Núcleo COL)

\begin{tabular}{|l|c|c|c|c|}
\hline $\begin{array}{c}\text { Universidad } \\
\text { del Zulia } \\
\text { (Núcleo COL) }\end{array}$ & Docentes & Estudiantes & Administrativo & Total \\
\hline $\begin{array}{c}\text { Programa de } \\
\text { Ingeniería }\end{array}$ & 36 & 4261 & 4 & 4301 \\
\hline \multicolumn{2}{|c|}{ Fuente: DITICLUZ (2008) } & Personal & \\
\hline
\end{tabular}

A su vez, la población estudiantil esta subdividida en tres (3) escuelas de ingeniería: Civil con 524 alumnos, de

Petróleo con 2075 alumnos, y Mecánica 
con 1662 alumnos (Cuadro 02).

Cuadro 02: División de las escuelas en el Programa de Ingeniería (Núcleo COL)

\begin{tabular}{|l|c|}
\hline \multicolumn{1}{|c|}{ Escuelas de Ingeniería (Núcleo COL) } & Estudiantes \\
\hline Ingeniería Civil & 524 \\
\hline Ingeniería Petróleo & 2075 \\
\hline Ingeniería Mecánica & 1662 \\
\hline
\end{tabular}

Fuente: $\quad$ DITICLUZ

(2008)

Para analizar con eficiencia una

población, se toma una parte de ella. A esto se le llama muestra. La muestra se

observa detenidamente y una vez logrado su estudio, se infieren resultados generales, lo cual significa que con la muestra se puede conocer a la población. Según Bavaresco (2001, p. 91), el muestreo aleatorio estratificado consiste en hacer un análisis previo de la población, que se divide en partes y luego se selecciona dicha muestra. En el caso del programa de Ingeniería de la Universidad del Zulia (Núcleo COL), la población estudiantil 
fue estratificada según se recoge en el

Cuadro 03.

Cuadro 03: Población estudiantil estratificada

\begin{tabular}{|c|c|c|c|}
\hline $\begin{array}{c}\text { Escuelas de Ingeniería (Núcleo } \\
\text { COL) }\end{array}$ & Estudiantes & Porcentaje de alumnos & $\begin{array}{c}\text { Cantidad a } \\
\text { encuestar }\end{array}$ \\
\hline Ingeniería Petróleo & 2075 & $49 \%$ & 179 \\
\hline Ingeniería Mecánica & 1662 & $39 \%$ & 44 \\
\hline Ingeniería Civil & 524 & $12 \%$ & $\mathbf{3 6 6}$ \\
\hline Total: & $\mathbf{4 2 6 1}$ & $\mathbf{1 0 0 \%}$ & \\
\hline
\end{tabular}

Fuente: Los Autores (2008)

En cuanto a las muestras de los docentes, estratifican porque la muestra total es que fue de 36 personas, y del personal mucho menor.

administrativo, con 4 personas, estas no se

Cuadro 04: Muestreo estratificado en la Universidad del Zulia, Núcleo COL

Fuente: Los Autores (2008)

\begin{tabular}{|c|c|c|c|}
\hline Población & Muestra & $\begin{array}{r}\text { Universida } \\
\text { Programa de }\end{array}$ & $\begin{array}{l}\text { del Zulia (Núcleo COL) } \\
\text { geniería } \\
\text { Muestreo }\end{array}$ \\
\hline \multirow{2}{*}{$\begin{array}{l}4301 \\
\text { Sujetos }\end{array}$} & \multirow{2}{*}{$\begin{array}{l}366 \\
\text { Sujetos }\end{array}$} & \multicolumn{2}{|l|}{$\begin{array}{c}\text { Estudiantes: } \\
366\end{array}$} \\
\hline & & $\begin{array}{l}\text { Docentes: } \\
36 \text { sujetos }\end{array}$ & $\begin{array}{c}\text { Personal Administrativos: } \\
4 \text { sujetos }\end{array}$ \\
\hline & & \multicolumn{2}{|c|}{ Total de la Muestra: 406} \\
\hline
\end{tabular}

Instrumento

Se aplicó la técnica de encuesta, a través de un cuestionario compuesto por 26 preguntas de elección múltiple, dentro de una escala de actitudes tipo
Likert para la población estudiantil, docente y administrativa. Esta técnica sirvió de complemento para el desarrollo del primer y segundo objetivo planteado en el estudio. 
Validez y confiabilidad del instrumento

Chávez (2007, p.193) afirma que la validez "es la eficacia con que un instrumento mide lo que pretende medir". A su vez, para Méndez (2001, p. 196), la validez "es el grado en que una prueba mide lo que se propone". En términos generales, se puede decir entonces que la validez del constructo es la que determina que la prueba mide lo que se pretende medir. En el caso de esta investigación, se realizó la validez discriminante como una de las escalas de actitudes tipo Likert. La misma constituye una de las pruebas potentes en el análisis de ítems. El poder discriminatorio de cada ítem se realizó en forma posterior al cálculo de los valores promedios para cada ítem, tanto en los grupos altos como bajos, por medio del programa estadístico SPSS
17. $Y$ en lo que se refiere a la confiabilidad, se obtuvo mediante la aplicación de una prueba piloto del instrumento a una muestra de veinte (20) sujetos de un universo similar al seleccionado para esta investigación. Dado que la confiabilidad es el grado de congruencia con que se realiza la medición de una variable, se utilizó el coeficiente Cronbach (alfa), que obtuvo una fiabilidad de 0,800 al aplicarse al test con 26 ítems de varias alternativas.

\section{ANÁLISIS Y DISCUSIÓN DE LOS RESULTADOS}

Para la aplicación del instrumento, se tomó una muestra de trescientos sesenta y seis (366) estudiantes, treinta y seis (36) docentes, y cuatro (4) integrantes del personal administrativo

para un total de 406 
encuestas aplicadas. Los resultados se exponen en el mismo orden que fueron aplicados, para lo cual se utilizó el software SPSS 17 (Statistic Package for Social Science) para Windows.

Para los análisis estadísticos, en este estudio, se echó mano de una gran variedad de técnicas, que englobaban los objetivos planteados para alcanzar la variable objeto de estudio, aplicándose, un método descriptivo, Anova y un t de student para muestras relacionadas, todo ello, tomando en consideración que de la variable Uso de las TIC en la Gestión del Conocimiento, se extraen dimensiones. Luego se realizó la estadística final, en la que se aplicó el método Anova para determinar el uso que tienen las dimensiones hacia la variable. A continuación, se presentan las tablas y gráficas, así como los análisis según sus indicadores y dimensiones.

Variable Uso de las TIC en la Gestión del Conocimiento

Dimensión Aplicabilidad de las TIC Indicador: Aplicabilidad de las TIC.

En el análisis de la operacionalización de la variable, se apreció que esta dimensión no posee indicador, motivo por el cual la dimensión Aplicabilidad de las TIC pasa a ser como el mismo indicador al cual se le aplicó el método descriptivo para el desarrollo del análisis, porque en él se procura definir las cualidades de un evento por medio de los valores percentiles, la tendencia central, la dispersión, la distribución y también se comparan medias (ver Cuadro 05). 


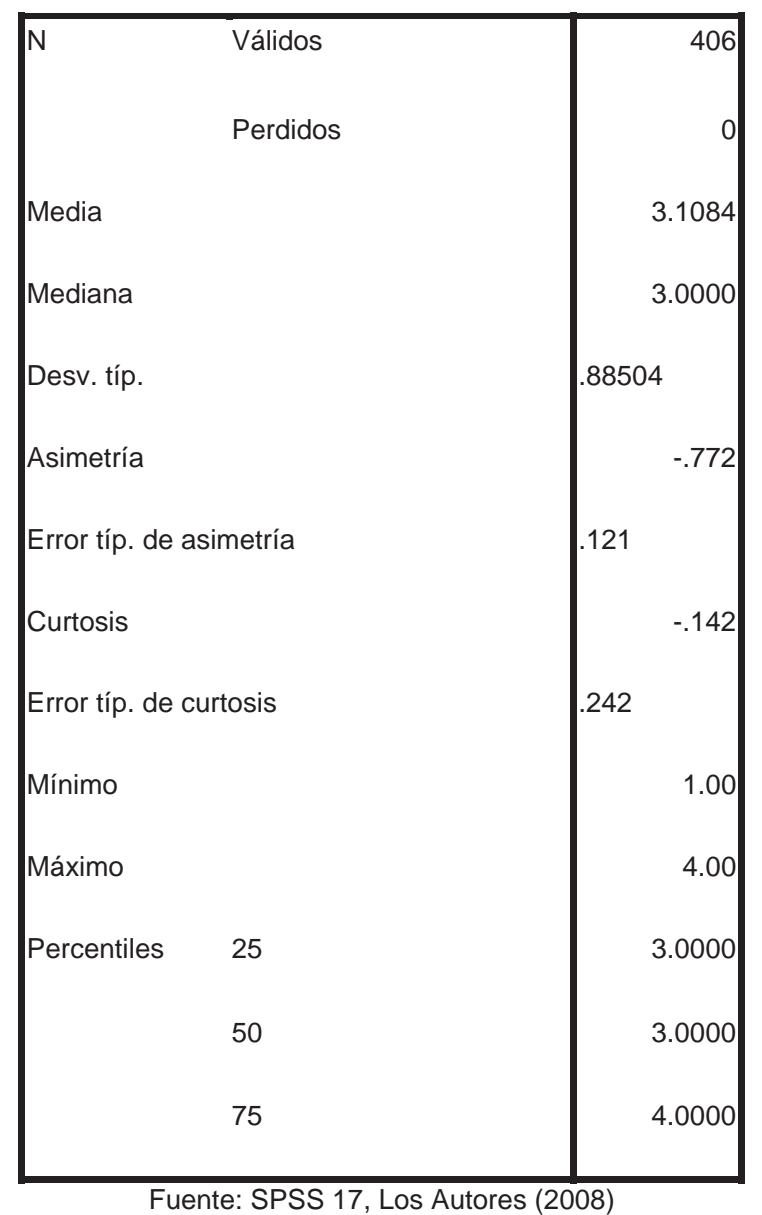

Se puede apreciar que para esta divide dos grupos con el mismo número dimensión no existen valores perdidos, de individuos. Por su parte, la dispersión lo que sugiere que todos los ítems indica la mayor o menor concentración fueron desarrollados, obteniéndose así de los datos con respecto a la media de un valor para la media de 3,1084 , centralización, que en este caso se siendo esta la suma de los valores evalúa con la desviación típica que fue dividido por el tamaño muestral. En esta de 0,88504 .

tabla, también se puede apreciar la mediana y la dispersión. La primera con un valor de 3,0000, el cual coincide con el percentil 50, que es el valor que

Pero, además, en el Cuadro 05, se puede apreciar la asimetría, que puede ser positiva o negativa en función del 
lado hacia donde se encuentre la cola de distribución. En este caso, se muestra que es de $-0,772$, motivo por el cual se dice que la concentración de los datos es a la derecha, y la Curtosis indica que la curva es más aplanada. Así, sobre esta dimensión, se puede concluir que se desvía un poco la concentración de los datos, indicando que no es un modelo normal, por presentar valores percentiles entre 3,0000 y 4,0000 . De todos modos, queda claro que un $25 \%$ sí hace uso de las TIC en los procesos de enseñanzaaprendizaje del programa de Ingeniería de la Universidad del Zulia (Núcleo $\mathrm{COL})$.

Estos resultados coinciden con lo planteado por Area (2001), quien propone que las experiencias llevadas a cabo en el uso e integración de las TIC en la enseñanza, permiten abordar de forma razonable la integración formal de estos medios en los distintos ámbitos educativos. Este proceso de integración e implantación de las TIC en los centros de enseñanza, y la creación de infraestructuras que facilitan el acceso a la sociedad de la información y las comunicaciones, se está llevando a cabo en un marco en el que se abordan paralelamente las siguientes actuaciones:

o Dotación de infraestructuras y equipamientos.

o Formación del profesorado en los planteamientos educativos de las TIC.

o Formación del alumnado y aprovechamiento educativo de las TIC.

o Impulso de los procesos de investigación e innovación.

o Promoción de las redes como soportes de contenidos educativos.

o Gestión académica y administrativa de los centros de enseñanza. 
Indicadores: Sistema de

Este análisis de Area (2001) ratifica que ese $25 \%$ de los encuestados sí aplica las TIC en los procesos de enseñanza aprendizaje, y el otro $75 \%$ se dedica a abordar paralelamente las actuaciones nombradas anteriormente. También se puede apreciar que su inclinación se acentúa hacia la dotación de la infraestructura y la formación del alumnado, mas no a la aplicabilidad de las TIC en todos los proceso de enseñanza.
Teleconferencia, Internet, Redes, Intranet, Estudios a Distancia, Audio, Video y Material Impreso

Con el objetivo de determinar si existen diferencias significativas entre los diversos indicadores que conforman la dimensión Recursos Tecnológicos, se aplicó el análisis de la varianza (ANOVA), el cual presentó un resultado de $\mathrm{F}=4,886$, asociado a un nivel de significancia de 0,000 , por lo que se establece que existen diferencias significativas entre ellos (Cuadro 06).

\section{Dimensión Recursos Tecnológicos}

Cuadro 06: ANOVA de un factor para la dimensión recursos tecnológicos Recursos Tecnológicos

\begin{tabular}{|l|r|r|r|r|l|}
\hline & $\begin{array}{c}\text { Suma de } \\
\text { cuadrados }\end{array}$ & GI & $\begin{array}{c}\text { Media } \\
\text { cuadrá } \\
\text { tica }\end{array}$ & F & Sig. \\
\hline Inter-grupos & 24,299 & 7 & 3,471 & 4,886 &, 000 \\
Intra-grupos & 2301,931 & 3240 &, 710 & & \\
Total & 2326,230 & 3247 & & & \\
\hline
\end{tabular}

Para ubicar las diferencias Duncan, la cual establece tres grupos significativas establecidas en Anova con diferencias significativas entre ellos. (Cuadro 07), se aplicó la prueba de El primer grupo, conformado por los 
indicadores Estudios a Distancias, Audio

e Internet; el segundo grupo, por Redes,

Intranet y Sistemas de Teleconferencia;

y el tercer grupo, por Video y Material

Impreso. El indicador menos favorecido es Estudios a Distancia, que presenta

un promedio de 3,0025, ubicándose dentro del baremo moderadamente alto. Esto significa que su rango está entre 2,5 y 3,25 .

Cuadro 07: Subconjunto Homogéneo para recursos tecnológicos

Recursos Tecnológico

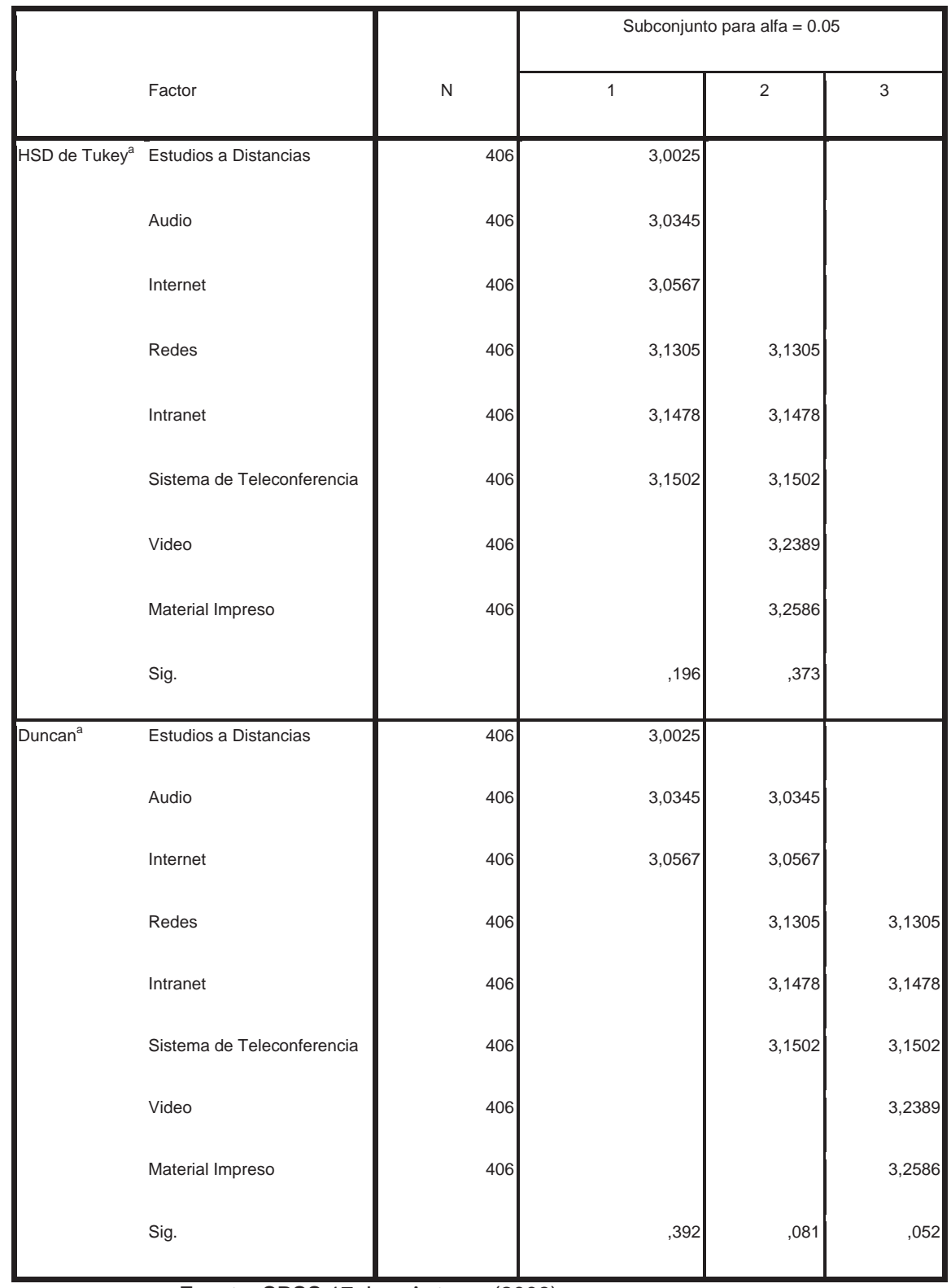

Fuente: SPSS 17, Los Autores (2008)

\section{Los indicadores Video y Material Impreso no presentan diferencias}


significativas con los dos grupos las encuestas, lo que explicaría el valor anteriores, por estar en un rango >0,05. en la significancia de 0,052 que alcanzó Ello a pesar de que en el baremo se este último grupo. En el siguiente grafico ubica como moderadamente alto, por de medias, se puede observar la tener un promedio de 3,2389 y 3,2586, variación que se presentó entre los respectivamente. Es decir, que las factores que intervienen en la dimensión estrategias utilizadas en la universidad Recursos Tecnológicos, donde se para estos dos indicadores son aprecia la división en tres grupos entre satisfactorias, según lo encontrado en los distintos indicadores analizados.

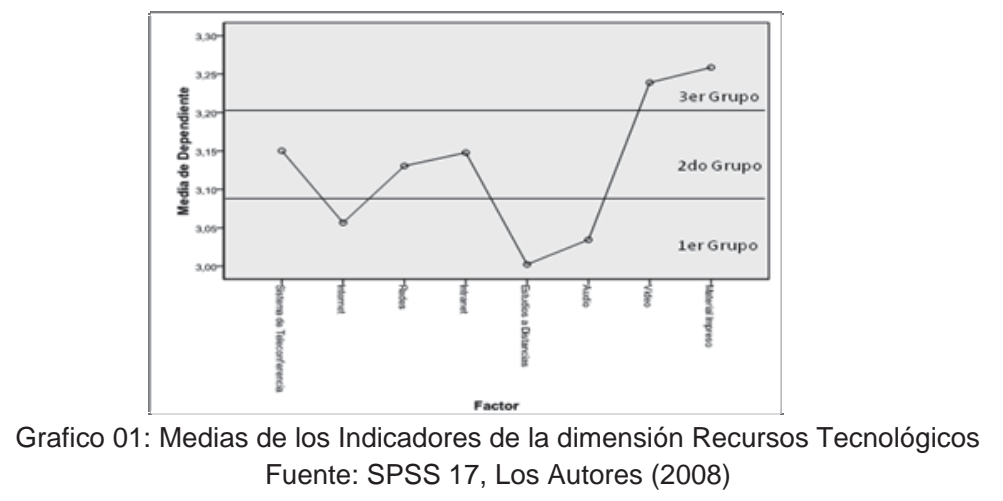

En la discusión de los resultados para esta dimensión, es necesario recordar lo planteado por Cebrían (1987), para quien el vídeo como recurso didáctico presenta una serie de características favorables, tales como su bajo coste o su facilidad de manejo, que le permiten estar presente en distintos momentos del proceso educativo, pues este puede ser: medio de observación, medio de expresión, medio de auto aprendizaje y medio de ayuda a la enseñanza.

El vídeo, entonces, es un medio didáctico que por sus posibilidades expresivas puede alcanzar un alto grado de expresividad, lo que hace de él una 
herramienta autónoma de aprendizaje con la que el alumno puede dominar un determinado contenido. Otro de los indicadores destacados en esta dimensión es el material impreso. Según lo citado por Chacón (1993), usado principalmente por quienes utilizan principalmente código verbales como palabras o textos $\mathrm{y}$, en menor grado, gráficas, dibujos, diagramas, fotografías, entre otros.

Es evidente reconocer que estos dos factores influyen de manera vertiginosa en el uso de la TIC para esta dimensión, por ser medios prácticamente que salen o se despliegan de la vieja educación, en donde el material impreso era uno de los elementos primordiales en la enseñanza, mientras que el video posee características propias de los medios de tecnología implementados desde sus inicios en la educación dirigida por las TIC.

Cuadro 08: ANOVA para la dimensión Infraestructura Tecnológica
Dimensión

Infraestructura

\section{Tecnológica}

Indicadores: Capacidad de Crecimiento Tecnológico, Portabilidad a otras Plataformas, Sistemas Operativos Administrativos, Sistemas Operativos de Aplicación, Tipos de Sistemas Operativos (Microsoft, Linux, entre otros.), Sistemas de Monitoreo y Control de Acceso, Ambiente Virtual de Aprendizaje, Equipos y Acceso a Internet.

Con el objetivo de verificar si existen diferencias significativas entre los diversos indicadores que conforman la dimensión Infraestructura Tecnológica, se empleó el análisis de la varianza (ANOVA), que presentó un resultado de $F=8,208$, asociado a un nivel de significancia de 0,000 , por lo que se establece que existen diferencias significativas entre ellos (Cuadro 08). 


\begin{tabular}{|l|r|r|r|r|r|}
\hline & $\begin{array}{r}\text { Suma de } \\
\text { cuadrados }\end{array}$ & Gl & $\begin{array}{r}\text { Media } \\
\text { cuadrática }\end{array}$ & F & Sig. \\
\hline Inter-grupos & 47,564 & 8 & 5,945 & 8,208 &, 000 \\
Intra-grupos & 2640,133 & 3645 &, 724 & & \\
Total & 2687,697 & 3653 & & & \\
\hline
\end{tabular}

Fuente: SPSS 17, Los Autores (2008)

Las diferencias significativas establecidas en Anova (ver cuadro 09) se obtuvieron mediante la aplicación de la prueba de Duncan, que establece cuatro grupos con diferencias significativas entre ellos. El primer grupo fue conformado por los indicadores:

Sistemas Operativos y Administrativos;

el segundo grupo, por tipos de Sistemas
Operativos (Microsoft, Linux, entre otros) y Capacidad de Crecimiento Tecnológico; el tercer grupo, por Sistemas de Seguridad de Monitoreo y Control de Acceso, Portabilidad a otras Plataformas, Sistemas Operativos de Aplicación y Acceso a Internet; y el cuarto grupo, por Equipos y Ambiente Virtual de Aprendizaje. El indicador menos favorecido en este caso fue el de Sistemas Operativos Administrativos, con un promedio de 2,9532, ubicándose en el baremo como moderadamente alto, lo que significa que su rango está entre 2,51-3,25.

Cuadro 09: Subconjunto Homogéneo para Infraestructura Tecnológica Infraestructura Tecnológica

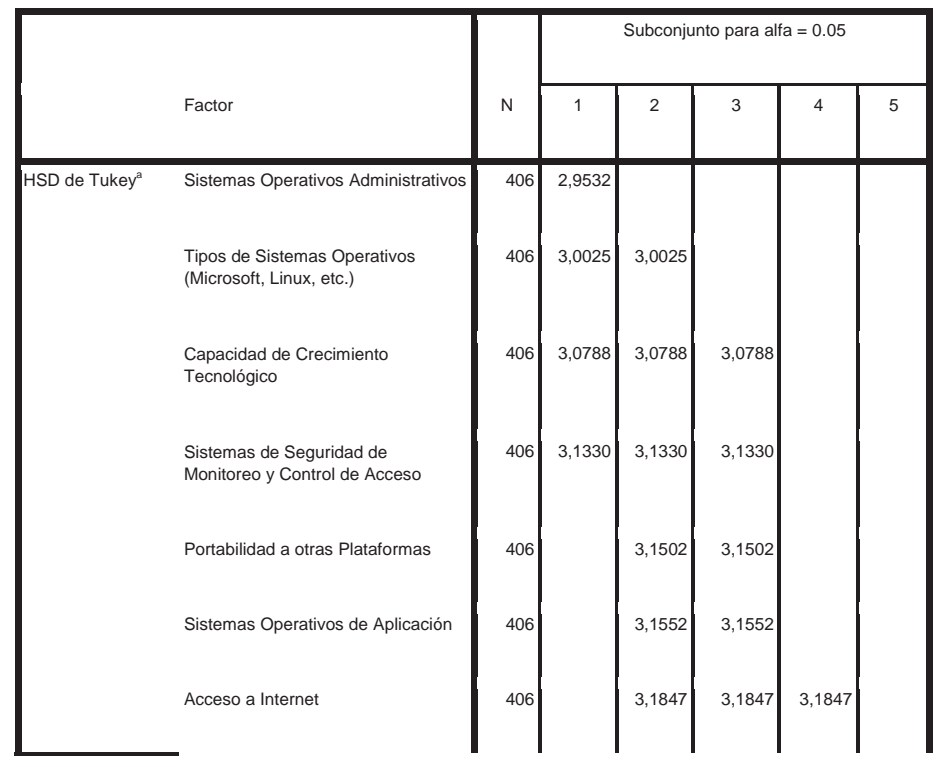




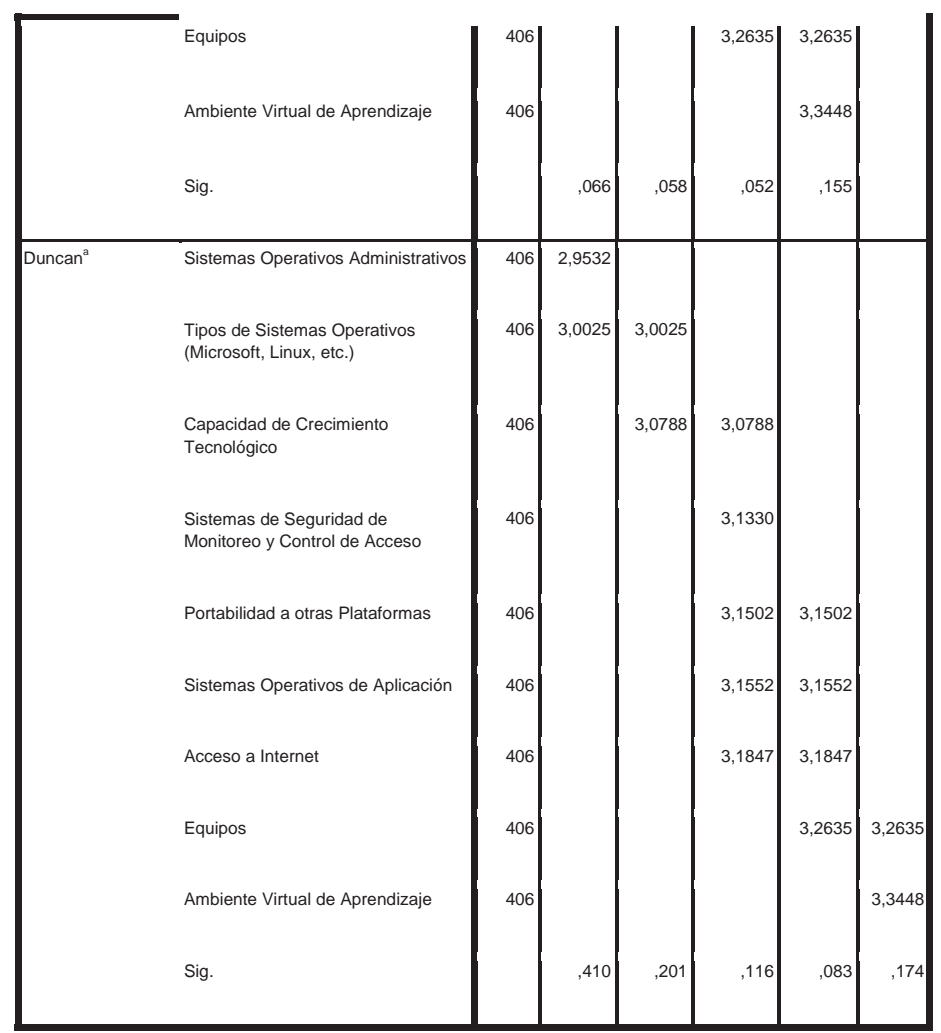

Fuente: SPSS 17, Los Autores(2008)

Entretanto, los indicadores Equipos y

Ambiente Virtual de Aprendizaje no

presentaron diferencias significativas en

relación con los tres grupos anteriores,

por estar en un rango $>0,05$ a pesar de

que en el baremo se ubica como

moderadamente alto, si se considera el

promedio de 3,2635 y 3,3448 ,

respectivamente. Es decir que las

estrategias que se aplican son

satisfactorias según los resultados

arrojados por el instrumento, lo que

explicaría un valor en la significancia de
0,174 para este último grupo.

El Gráfico 02 de medias muestra la variación que existe entre los 9 factores indicadores que intervienen en esta dimensión. Se aprecia la división en grupos de los distintos métodos aplicados, con una media dependiente para los casos analizados.

Grafico 02: Medias de los Indicadores de la dimensión Infraestructura Tecnológica 


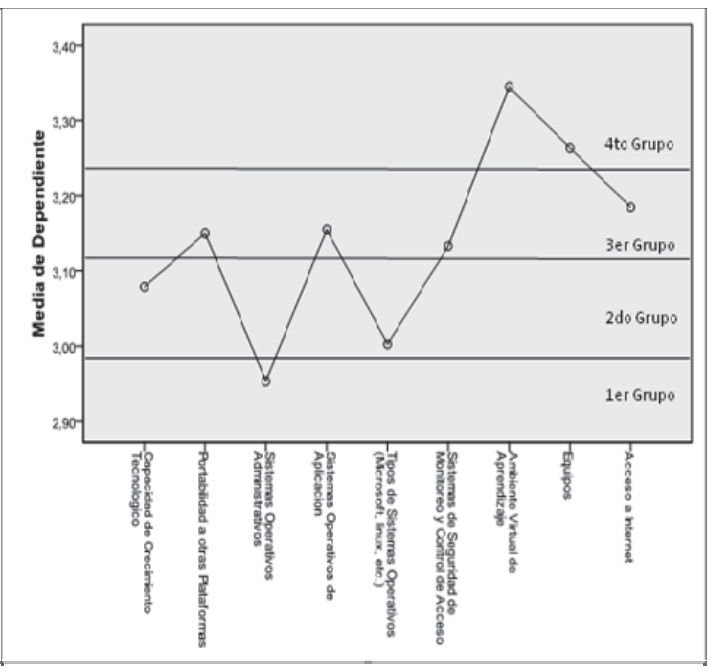

Fuente: SPSS 17, los Autores (2008)

A estas alturas, es imprescindible recordar lo planteado en el marco teórico por Gil (1997), quien propone que los equipos tecnológicos educativos complementan, refuerzan, adecuan la docencia actual y constituyen herramientas que facilitan tanto la enseñanza como el aprendizaje. Es indiscutible que, para crear esta situación educativa, el docente necesita de equipos tecnológicos $y$, en consecuencia, se debe ampliar la cobertura de estos. Por otro lado, los contextos de Ambiente Virtual de Aprendizaje se entienden como el resultado de organizar, en el tiempo y en el espacio, elementos como el contenido, la interacción, la evaluación, el seguimiento y la orientación, con el propósito de lograr el aprendizaje. A tono con lo anterior, Rivera (1997) considera que la expresión organizar exige definir cuál será el papel de cada elemento y cómo se relacionan entre sí en espacio y en tiempo, y que la denominación ambiente virtual se aplica cuando tal organización se interroga la manera cómo se han concebido los elementos de los ambientes existentes y, por tanto, identificando su cuestión fundamental y proponiéndole cambios significativos.

Es propicio reconocer que, en esta dimensión, los indicadores con mayor promedio son parte fundamental en los procesos de diseño de una Infraestructura Tecnológica, porque uno conlleva al otro. De modo que si hay implementación de equipos tecnológicos, a su vez, debe haber un 
Ambiente Virtual de Aprendizaje donde el alumno se sienta en óptimas condiciones de enseñanza para adquirir el conocimiento de una forma satisfactoria.

Dimensión Manejos de las TICIndicadores: Manejos de Enseñanza-Aprendizaje, Material Didáctico Implementado y Cultura Educativa

Con el propósito de conocer si existen diferencias significativas. entre los diversos indicadores que conforman la dimensión Manejos de las

TIC, se aplicó el análisis de la varianza (ANOVA). Este arrojó un resultado de $\mathrm{F}=14,004$, asociado a un nivel de significancia de 0,000 , por lo que se establece que existen diferencias significativas entre ellos (Cuadro 10).

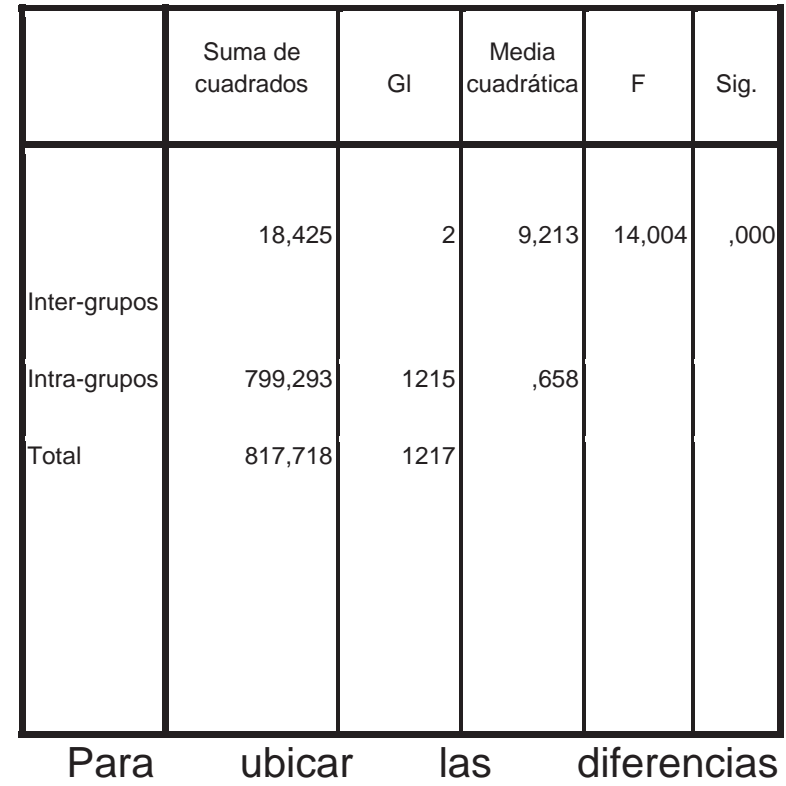

significativas presentadas en Anova, (ver Cuadro 11) se aplicaron las pruebas de Tukey y Duncan, las cuales mostraron dos grupos con diferencias significativas entre ellos. El primer grupo fue conformado por los indicadores Cultura Educativa y Material Didáctico 
indicador menos favorecido para esta Enseñanza-Aprendizaje con un promedio de 2,9655, ubicándose en

Cuadro11. Manejos de las TIC

\begin{tabular}{|c|c|c|c|c|}
\hline & \multirow[b]{2}{*}{ Factor } & \multirow[b]{2}{*}{$\mathrm{N}$} & \multicolumn{2}{|c|}{$\begin{array}{c}\text { Subconjunto para alfa }= \\
0.05\end{array}$} \\
\hline & & & 1 & 2 \\
\hline \multirow[t]{4}{*}{$\begin{array}{l}\text { HSD de } \\
\text { Tukey }^{a}\end{array}$} & $\begin{array}{l}\text { Manejos de Enseñanzas- } \\
\text { Aprendizaje }\end{array}$ & 406 & 2,9655 & \\
\hline & Cultura Educativa & 406 & & 3,1724 \\
\hline & $\begin{array}{l}\text { Material Didáctico } \\
\text { Implementado }\end{array}$ & 406 & & 3,2586 \\
\hline & Sig. & & 1,000 & ,284 \\
\hline \multirow{4}{*}{ Duncan $^{a}$} & $\begin{array}{l}\text { Manejos de Enseñanzas- } \\
\text { Aprendizaje }\end{array}$ & 406 & 2,9655 & \\
\hline & Cultura Educativa & 406 & & 3,1724 \\
\hline & $\begin{array}{l}\text { Material Didáctico } \\
\text { Implementado }\end{array}$ & 406 & & 3,2586 \\
\hline & Sig. & & 1,000 & , 130 \\
\hline
\end{tabular}

beremo como moderadamente alto, lo que significa que su rango se halla entre 2,51 y 3,25 .

En cambio, el indicador del segundo grupo, Manejos de Enseñanza- dimensión fue el de Manejos de Aprendizaje, no presentó diferencias significativas en comparación con los del primer grupo, por estar en un rango $>0,05$, a pesar de que en el baremo se ubica como moderadamente alto al presentar un promedio de 3,1724 y 3,2586, respectivamente. Esto demuestra que las estrategias se aplican de forma satisfactoria en la universidad, según los resultados arrojados en la encuesta, y eto explica un valor en la significancia de 0,130.

En el Gráfico 03, se aprecia de una forma clara cómo intervienen los tres (3) factores que conforman esta dimensión: un grupo llamado Manejos de Enseñanza-Aprendizaje y otro grupo que se denominó Material Didáctico Implementado y Cultura Educativa. 


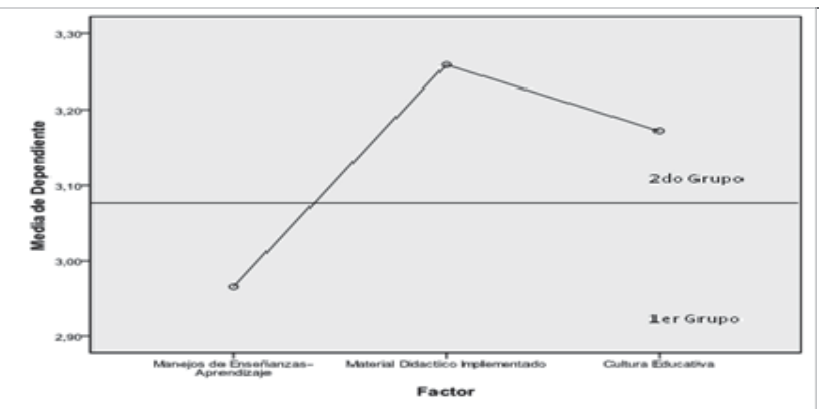

Grafico 03: Medias de los Indicadores de la dimensión Manejos de las TIC

Fuente: SPSS 17, Los Autores (2008)

Según Ausubel (1996), es urgente un cambio en la cultura educativa para romper viejos esquemas y paradigmas sobre las maneras tradicionales de hacer docencia, investigación y extensión, así como tomar en consideración el factor humano y los valores fundamentales del hombre como elemento clave en todo proceso de cambio.

Por otra parte, Chevallard (1991) plantea que los materiales didácticos se han elaborado expresamente para trabajar con ellos en el proceso de enseñanza-aprendizaje: un mapa, el globo terráqueo, y en el campo de las TIC: software educativos, juegos interactivos, webquest, entre otros. Teniendo en cuenta esta distinción, se debe especificar que el material didáctico propiciado por las TIC constituye un tipo de texto especializado en el que intervienen distintos actores que portan saberes específicos, más aún que en otro tipo de material didáctico. Se trata de transponer, empleándolos, un texto científico o artístico conocimiento erudito a un texto instructivo conocimiento a enseñar en el que el propósito no es sólo comunicar un contenido, sino enseñar un contenido a otro.

Después de mencionar a los autores anteriores como parte de la discusión de los resultados, es preciso analizar sus planteamientos en la intervención de la Cultura Educativa. Al respecto, en el campo de la educación, los procesos educativos necesitan un verdadero cambio para enfrentar las dificultades de esta era de globalización. La implantación de los materiales 
didácticos, que son un trampolín a la hora de incorporar nuevas culturas educativas en los ámbitos de enseñanza, presentan características propias, que promueven la unión de materiales tradicionales, y materiales didácticos tecnológicos.

Dimensión Necesidad de las TIC Indicador: Necesidad del Uso de las TIC en la Gestión del Conocimiento.

En esta dimensión se puede visualizar que existe un solo indicador, motivo por el cual se emplea el método descriptivo para el proceso del análisis que involucra la tendencia central, la dispersión, la distribución y también se comparan las medias.
Se puede apreciar que para esta dimensión, no existen valores perdidos, lo que demuestra que todos los ítems fueron desarrollados, obteniéndose un valor para la media de 3,1798 , siendo esta la suma de los valores divididos por el tamaño muestral (ver Cuadro 12). En esta tabla, también se puede apreciar la mediana, con un valor de 3,0000 el cual coincide con el percentil 50, que es el valor que divide dos grupos con el mismo número de individuos. $\mathrm{Y}$ en cuanto a la dispersión que es la que indica la mayor o menor concentración de los datos con respecto a la media de centralización, e en este caso se evalúa con la desviación típica, que es 0,81023 . 


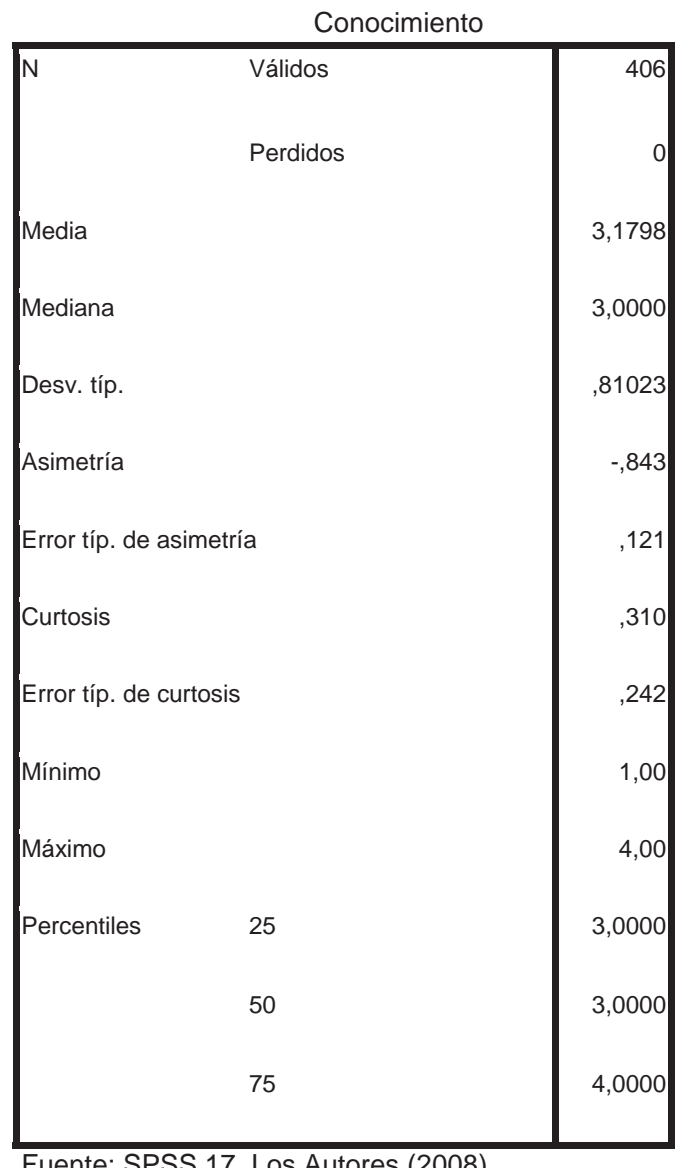

Fuente: SPSS 17, Los Autores (2008)

En el Cuadro 12, también se puede apreciar la asimetría, que indica si es positiva o negativa según el lado en que se encuentra la cola de la distribución, en este caso se muestra que es de 0,843, motivo por el cual se puede decir que la concentración de los datos es a la derecha, y la Curtosis indica que la curva es mas aplanada. La discusión de los resultados de esta dimensión permite concluir que se desvía un poco la concentración de los datos, lo que indica que no es un modelo normal, por presentar valores percentiles entre 3,0000 y 4,0000. Así, hay una tendencia de datos arrojados por el instrumento de un $75 \%$ que sí tiene Necesidad de las TIC en los procesos de enseñanzaaprendizaje del programa de Ingeniería en la Universidad del Zulia (Núcleo $\mathrm{COL})$.

Estos resultados coinciden con lo planteado por Collins (1998), quien expresa que el potencial en las tecnologías digitales, las cuales albergan una firmeza social reconocida de la necesidad de utilizar las TIC para apoyar los procesos de enseñanzaaprendizaje, exige a las instancias responsables de formar docentes hacerse cargo del tema, dado que estos escenarios representan nuevos desafíos que la educación debe abordar y para los cuales los docentes en formación deberían estar preparados. La inserción de las TIC en los contextos educativos 
puede reportar beneficios para el sistema educativo en su conjunto: alumnos, docentes y la comunidad educativa en general. En el caso de los docentes, las tecnologías ponen a su disposición diversos recursos digitales: software, documentos, página web, entre otros; facilitan, además, la participación en redes de docentes y apoyan el trabajo de proyectos en forma colaborativa con otros centros educativos.

aprendizaje, posee recursos para enfrentar nuevos desafíos que la educación debe abordar y para los cuales los docentes y estudiantes en formación deberían estar preparados. Por el contrario, el otro $25 \%$ abordan los procesos de enseñanza de forma tradicional, lo que implica que no tienen ningún tipo de necesidad de implementar en sus procesos de enseñanza de las TIC.

Dimensión Apoyo Institucional a las TIC en la Gestión del Conocimiento

Este análisis citado por Collins (1998) ratifica que ese $75 \%$ de los encuestados, que sí tiene Necesidad de las TIC en los procesos de enseñanza

Indicadores: El Rol del Docente e Intervención de los Elementos Pedagógicos. 


\begin{tabular}{|l|r|r|r|r|}
\hline & & & & \\
& Media & $\mathrm{N}$ & $\begin{array}{c}\text { Desviación } \\
\text { típ. }\end{array}$ & $\begin{array}{c}\text { Error típ. } \\
\text { de la } \\
\text { media }\end{array}$ \\
\hline Par 1 $\begin{array}{l}\text { El rol del } \\
\text { Docente } \\
\text { Intervención de } \\
\text { los Elementos } \\
\text { Pedagógicos }\end{array}$ & 3.2107 & 261 & .62677 & .03880 \\
\hline
\end{tabular}

Fuente: SPSS 17, Los Autores (2008)

Como hemos venido haciendo a lo largo de la exposición y análisis de los resultados, con el propósito de establecer si existen diferencias significativas entre los indicadores que conforman la dimensión Apoyo Institucional a las TIC en la Gestión del Conocimiento, se aplicó un recurso estadístico, en este caso la t de Student para muestras relacionados (ver Cuadro 14), la cual arrojó una $t=0,997$, asociada a un nivel de significancia de 0,320 , por lo que se establece que no existen diferencias significativas entre ellas. Ambos indicadores, el Rol del Docente y la Intervención de los Elementos Pedagógicos alcanzan una media de 3,2759 y 3,2107 , respectivamente. Ambos indicadores se ubican dentro del baremo en un nivel alto, lo que indica que los resultados en la encuesta son los más altos en todos los análisis.

Cuadro 14: Prueba T de Student para muestras relacionadas en la dimensión Apoyo Institucional a las TIC en la Gestión del Conocimiento

Prueba de muestras relacionadas

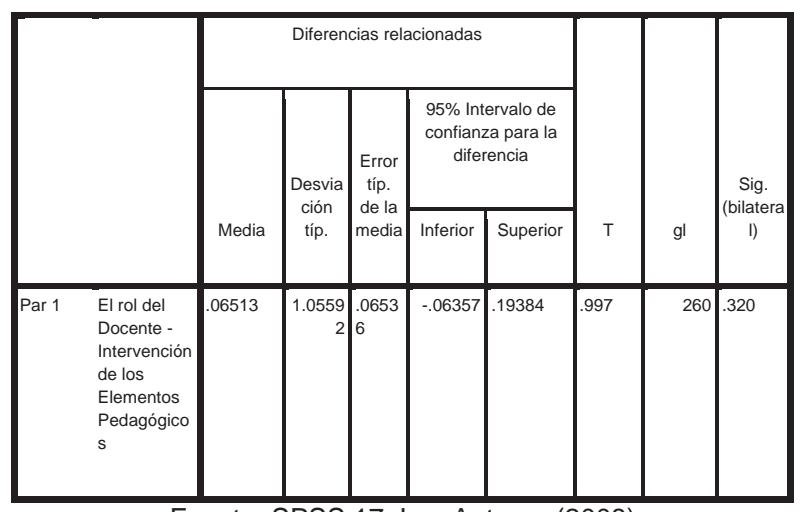

Fuente: SPSS 17, Los Autores (2008)

Los resultados arrojados por la encuesta

demuestran que esta es la dimensión 
con mayor promedio de satisfacción en

todo el estudio, por la mínima diferencia que existe entre sus indicadores, como es el caso de El rol del Docente y la Intervención de los Elementos Pedagógicos. Para profundizar el tema es necesario la opinión de Chacón (1994), quien cita lo siguiente: "Las universidades deben estar a la vanguardia de los cambios que se suscitan, rediseños de los procesos combinados con la tecnología informática, siendo este uno de los caminos más atractivos para la oxigenación de las universidades u organizaciones educativas".

La fortaleza de las universidades en el terreno de las TIC, en la docencia, es el profesorado y el conocimiento. Pero Después de haber analizado todos los ítems por separado en sus respectivas dimensiones, ahora se procede a evaluar las dimensiones para la variable Uso de las TIC en la Gestión del Conocimiento en la Universidad del ninguna universidad es fuerte en todos los campos. Se hace imprescindible la alianza entre las instituciones, la formación de equipos. El compromiso y el apoyo institucional a este tipo de experiencias es un elemento primordial, tanto para la supervivencia de las mismas como para la evolución de las entidades universitarias. Pero, así como se ve incierto el futuro de las iniciativas particulares de los profesores, tampoco parece que puedan tener éxito proyectos impuestos desde los órganos de gobierno. Por esta razón, es necesario concienciar a la comunidad universitaria. Junto al apoyo institucional, se considera, por lo tanto, elemento crucial el convencimiento del profesorado como factor clave del éxito Zulia (Núcleo COL). Con el objetivo de saber si existen diferencias significativas entre las diversas dimensiones que conforman esta variable, se aplicó el análisis de la varianza (ANOVA), el cual presenta un resultado de $\mathrm{F}=2,182$, 
asociado a un nivel de significancia de 0,054, por lo que se establece que existen diferencias significativas entre ellas (Cuadro 15).

Cuadro 15: ANOVA de un factor para el promedio de las Dimensiones Dependiente

\begin{tabular}{|l|r|r|r|r|r|}
\hline & $\begin{array}{r}\text { Suma de } \\
\text { cuadrado } \\
\text { S }\end{array}$ & Gl & $\begin{array}{r}\text { Media } \\
\text { cuadrática }\end{array}$ & F & Sig. \\
\hline Inter-grupos & 3,914 & 5 &, 783 & 2,182 &, 054 \\
Intra-grupos & 871,578 & 2430 &, 359 & & \\
Total & 875,492 & 2435 & & & \\
\hline
\end{tabular}

Fuente: SPSS 17, Los Autores (2008)

Para ubicar las diferencias significativas establecidas en Anova (ver Cuadro 16), se aplicó la prueba de Duncan, por la cual se establecieron dos grupos con diferencias significativas entre ellos. El primer grupo fue conformado por las dimensiones Aplicabilidad de las TIC, Recursos Tecnológicos, Manejos de las TIC e Infraestructura Tecnológica; y el segundo grupo, por Necesidad de las TIC y Apoyo Institucional a las TIC en la Gestión del Conocimiento. La dimensión menos favorecida en este primer grupo es Aplicabilidad de las TIC, por presentar un promedio de 3,1084, ubicándose dentro del baremo como moderadamente alta, lo que significa que su rango se halla entre 2,51-3,25.
Cuadro 16: Subconjunto Homogéneo del promedio de las Dimensiones

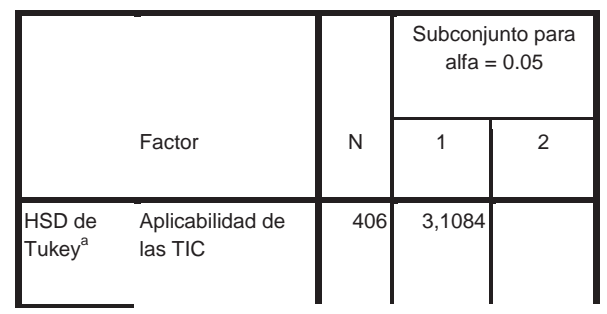




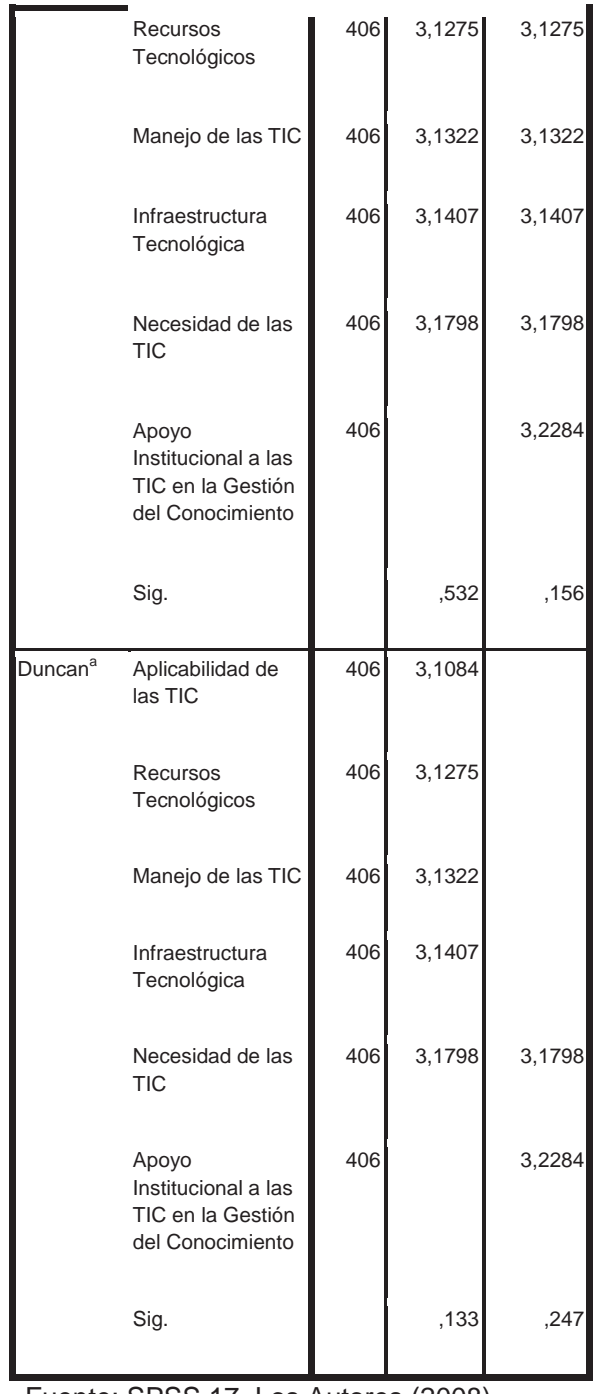

Fuente: SPSS 17, Los Autores (2008)

En cambio, la dimensión Apoyo Institucional a las TIC en la Gestión del Conocimiento no presenta diferencias significativas con el segundo grupo, porque

Por el contrario, resulta evidente y curioso el bajo índice que presenta la en el baremo se ubica como moderadamente alta. Tales resultados muestran que esta dimensión se aplica de forma satisfactoria según lo encontrado en las encuestas, lo que explicaría su valor en la significancia, que fue de 0,247.

En el Gráfico de Medias 04, se aprecia que la dimensión Apoyo Institucional a las TIC en la Gestión del Conocimiento presenta la media más alta de todas las dimensiones presentes en este estudio.

Grafico 04: Medias de las Dimensiones presente en este estudio

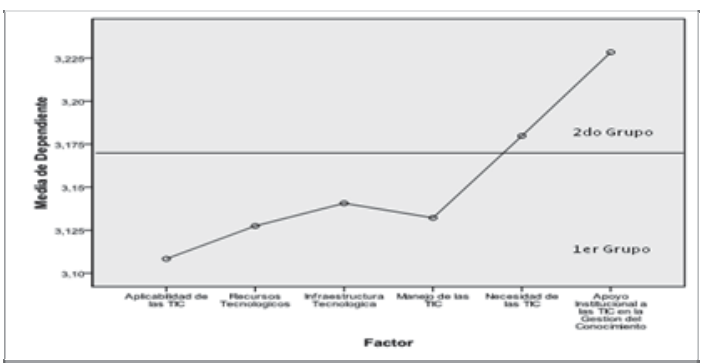

Fuente: SPSS 17, Los Autores (2008)

dimensión aplicabilidad de las TIC en relación con el resto de las 
dimensiones. Este resultado refleja que solo el $25 \%$ de la población aplica las TIC en sus procesos de enseñanza, pero lo contradictorio es que el resto de las dimensiones, como por ejemplo la antes citada, Apoyo Institucional a las TIC en la Gestión del Conocimiento, presenta el promedio más alto dentro del rango que se evalúa.

Pero en realidad estos resultados no son tan alarmantes como se muestra, porque si se visualiza el rango de media dependiente, se puede ver que la diferencia entre ambas no es tan sorprendente. En efecto, al analizar estos rangos se aprecia que se mantienen entre 3,10 y 3,225 , es decir, que se mantiene en un rango elevado, porque si se recuerda la escala que se utilizo aquí fue de 1 a 4 . Por lo tanto, esta investigación presenta una gran fiabilidad a la hora de evaluar la variable de estudio.

Una vez finalizado el análisis y discusión de los resultados obtenidos de la aplicación del instrumento a estudiantes, docentes y personal administrativo del programa de Ingeniera de la Universidad del Zulia (Núcleo COL), se procede a la discusión de los mismos en cuanto a los objetivos específicos planteados en esta investigación. También se comparan los resultados con las proposiciones resaltadas en el marco teórico por parte de los distintos autores considerados. Todo ello con el objeto de establecer las tendencias que se generan y los elementos necesarios para comparar el uso actual con el patrón de referencia.

En relación al objetivo específico consistente en "determinar el uso de las TIC en la gestión del conocimiento en la universidad del Zulia (Núcleo 
COL)", los datos aportados por el instrumento revelaron que los alumnos, docentes y personal administrativo poseen conocimientos sobre el uso de las TIC en la gestión del conocimiento, y tienen claro que el uso de las TIC refuerza, fortalece, mejora y complementa el material de aprendizaje en el desarrollo de acciones educativas, corrigiendo debilidades en el estudiante.

En cuanto al objetivo de "identificar los patrones de referencia en cuanto al uso de las TIC en la gestión del conocimiento en la Universidad del Zulia (Núcleo COL)", se encontró, por medio de la encuesta, que los patrones de referencia como los de Gil (1997) y Knowledge (2002), se mantienen a la vanguardia, permitiéndole al estudiante considerar esos patrones como una herramienta de ayuda en las enseñanzas de las nuevas tecnologías, a través de la interacción.

\section{CONCLUSIONES}

Los resultados obtenidos permitieron llegar a las siguientes conclusiones:

En relación al primer objetivo específico propuesto en la investigación, "determinar el uso de las TIC en la gestión del conocimiento en la universidad del Zulia (Núcleo COL)", se concluye que los alumnos implementan en gran medida el uso de las herramientas tecnológicas, debido a la necesidad que tienen hoy día de utilizar las TIC para mejorar su rendimiento académico. Más en concreto, se evidenció, por parte de docentes y alumnos, que el aprendizaje y conocimiento de las nuevas tecnologías de información y comunicación en la gestión del 
conocimiento, se refleja en el alto valor alcanzado por la dimensión Apoyo Institucional a las TIC en la Gestión del Conocimiento.

En relación con el objetivo de "identificar los patrones de referencia en cuanto al uso de las TIC en la Gestión de Conocimiento en la Universidad del Zulia (Núcleo COL)", se concluye que se identifican los patrones de referencia en el uso de las TIC, como el de Gil (1997), quien consideró en su libro, Sistemas y tecnologías de la información para la gestión, que hay un ciclo de audacia en el hecho de combinar los sistemas y las tecnologías de información en un proceso de gestión, en cualquier etapa de una organización. El otro patrón considerado fue el de Knowledge (2002), en su libro Gestión del conocimiento, en el cual estableció que el proceso de identificación, optimización y gestión dinamiza los activos intelectuales, convirtiéndolos en una forma de conocimiento explícito o tácito poseído por personas o comunidades.

Con respecto al tercer objetivo, "comparar el uso actual con el patrón de referencia", se aprecia de manera clara que las referencias bibliográficas que apoyaron esta investigación permiten a los investigadores comparar ese patrón con el uso actual de las TIC en la Universidad del Zulia (Núcleo $\mathrm{COL})$, por lo cual se demostró que la mayor eficiencia en aplicar el uso de las TIC en la gestión del conocimiento fue en la dimensión Apoyo Institucional a las TIC en la Gestión del Conocimiento, por obtener un promedio de 3,2284 en su rango de 1 a 4. Este valor se toma como alto dentro del ese rango.

Finalmente, en función al objetivo 
general de la investigación, se puede concluir que los resultados fueron en gran parte satisfactorios.

\section{RECOMENDACIONES}

Una vez concluida la investigación y a partir de los resultados obtenidos, sus autores sugieren:

- Desarrollar la infraestructura tecnológica de telecomunicaciones y de conectividad a Internet para que sea utilizada por los actores del proceso educativo de una manera más efectiva dentro de los salones de clase en el programa de Ingeniería de la Universidad del Zulia (Núcleo COL).

- Continuar analizando la situación actual del proceso de aprendizaje en las diferentes unidades curriculares que componen las carreras, para incorporar las nuevas tecnologías, y superar así las debilidades que se puedan presentar en estos temas en la Universidad del Zulia (Núcleo COL).

- Desarrollar contenidos educativos en formato electrónico para diferentes niveles y modalidades del sistema educativo universitario del venezolano a nivel general.

- Incorporar cátedras virtuales y usar las TIC para fortalecer la educación universitaria, rigiéndose por los pasos que integran la gestión del conocimiento.

- Proponer planes de formación permanente para garantizar la capacitación y puesta al día en el uso de las tecnologías como entorno y herramienta de información en las instalaciones de la Universidad del Zulia (Núcleo COL). 
Bavaresco, A. (2001). Proceso

- Seguir fomentando el apoyo institucional en la Universidad del Zulia, para que el programa de Ingeniería se posicione en el mayor rango entre los estudios similares a este.

\section{REFERENCIAS}

Área, M. (2001). La oferta de educación superior a través de Internet. Análisis de los campos virtuales de las universidades españolas, La Laguna. Artículo publicado en: Revista Española de Pedagogía, Vol. 63, № 230, 2005, págs. 85-100, ISSN 0034-9461
Metodológico de la Investigación.

Caracas: Academia Nacional de la Ciencia.

Bueno, C. (2003). Gestión del conocimiento en las universidades y organismos públicos de Madrid. Edita Dirección General de Investigación, Consejería de Educación, Comunidad de Madrid Maquetación Cromotex. Impresión: Elecé Industria Gráfica. Depósito Legal M$54391-2003$

Brooking, A. (1997). El capital

Ausubel, D. (1996). Psicología Educativa. Un punto de Vista Cognoscitivo. México: Editorial Trillas. intelectual, el principal activo de las empresas del tercer milenio. Barcelona: Editorial Paidos.

Cebrián, M. (1987) El Vídeo Educativo. 
En Actas del II Congreso de Tecnología Educativa. Madrid: Sociedad Española de Pedagogía. Tomado de: Bravo Ramos, J.L. ¿Qué es un video educativo? ICE de la Universidad Politécnica de Madrid.

URL http://www.ice.upm.es/wps/jlb r/Documentación/QueEsVid.pdf educación a distancia. Conferencia magistral de la Primera Reunión Latinoamericana a Distancia de Educación Superior Abierta y a Distancia, Disponible en: www.anep.edu.uy/webct/oferta_ed uc/exp_contenidos/swf/pg/lect/14/L ectura5.doc. Consultado el 3 de febrero de 2009.
Collins, A. (1998). El potencial de las tecnologías de la información para la educación. En: Vizcarro, C., y León, J. Nuevas Tecnologías para el aprendizaje, pp. 29-46. Madrid: Pirámide.
Chávez, N. (2007). Introducción a la Investigación educativa. Maracaibo: La Columna.
Chevallard, (1991). La transposición didáctica: del saber sabio al saber enseñado. Editorial AIQUE Grupo Editor.
Chacón, F. (1994). Un modelo de evaluación de los aprendizajes en
DITICLUZ. (2008) Dirección de Información de Tecnología de 
Información y Comunicación de la Universidad del Zulia. Ciudad: Maracaibo - Venezuela. Es un Departamento de Dirección de Información y Comunicación de la Universidad del Zulia. (Suministro la Data Estadística).

Gil, I. (1997). Sistemas y tecnologías de la Información para la Gestión. España: McGraw - Hill / Interamericana de España, S.A.U.

Joyanes, L., (1998). Cibersociedad.

Los Restos sociales ante un nuevo Mundo Digital. Bogotá: McGraw Hill

Knowledge, M. (2002). Gestión del Conocimiento. DAEDALUS - Data, Decisions and Lenguaje, S.A. (Versión Electrónica). La Habana.

Información y Comunicación para de enero de 2009] Disponible en: Desarrollo. [Fecha de consulta: 04 Méndez, C. (2001). Diseño $y$ Desarrollo de un Proceso de Investigación. España: McGraw Hill. Editorial Nomos S.A.

Rivera, E. (1997). Aprendizaje Asistido por Computadora. Diseño y realización. Guía para la asignación Fundamentos de Informática Educativa. Maracaibo - Venezuela. Maestría Informática Educativa. (TESIS)

Nishio, M. (2004). Tecnologías de http://www.listin.com.do//cuerpos7 
Nonaka, I., (1994). Dynamic Theory of Organizational Knowledge Creation. Organization Science, Vol. 5, n 1 (Febrero), pp.14.37.

Nonaka, I. y Takeuchi, H. (1995). La organización creadora de conocimiento: Cómo las compañías Japonesas crean la dinámica de la innovación. México: Oxford Press. de CVOrganization Science, Vol. 5, $\mathrm{n}^{\circ} 1$ (Febrero), pp.14.37.

Villarreal, S. (2007). Introducción a la computación. México: McGraw-Hill 